\title{
"Simple" diagnostics for characterization of low-pressure chemically active plasmas*
}

\author{
D. K. Otorbaev \\ Kyrgyz National Academy of Sciences, Chui prospect 265A, Bishkek, 720071, \\ Kyrgyz Republic
}

\begin{abstract}
In the current paper, the "simple" diagnostics for characterization of nonequilibrium chemically active plasmas are discussed. As to experimental methods, "simple" noninvasive spectroscopic methods of ionizing and recombining plasma characterization have been emphasized. The word "simple" in the title is presented in quotes, which means that the methods are assumed to be simple only conditionally. In order to be sure that the measurements indeed provide correct information on the plasma parameters, a detailed understanding of kinetics of each particular plasma source is required. Moreover, the validity of spectroscopic methods should be checked by independent diagnostic techniques.
\end{abstract}

\section{INTRODUCTION}

Understanding the chemistry of nonequilibrium plasmas and optimization of the plasma sources requires determination of densities of the species presented: atoms, molecules, radicals, ions and electrons-preferably spatially defined, with their energy distributions-velocities, and internal states.

With the appearance of sophisticated experimental techniques for characterization of nonequilibrium plasmas, the quality of diagnostic methods, and as a consequence the quality of the experimental results, improves very rapidly. Quite often, new discoveries in plasma applications are based on rather original diagnostic methods. Equipment based on newly opened phenomena, for example, the methods used various laser spectroscopy techniques, clearly brings new in-depth knowledge on the internal parameters of plasmas. However, everything has a price. Invention and following application of new diagnostic techniques require quite significant investments. Not all university and application-oriented research laboratories can afford the implementation of the newest state-of-the-art experimental technologies. Traditional plasma diagnostic techniques are still very actively used [1]. However, the are not always used correctly.

In this paper, the "simple" noninvasive spectroscopic methods for the characterization of lowpressure chemically active plasmas are discussed. Illustrations are given on an example of the diagnostics of two main types of laboratory plasmas-ionizing and recombining.

\section{IONIZING PLASMAS}

One of the most complicated problems in diagnostics of chemically active plasmas is knowledge of its real chemical composition. Glow discharges at reduced pressures in molecular gases represent typical examples of nonequilibrium plasma with a complex chemical composition [2]. High-energy inputs to such discharges results in fast reactions in the plasma, and unpredictable distribution of their compo-

*Lecture presented at the $15^{\text {th }}$ International Symposium on Plasma Chemistry, Orléans, France, 9-13 July 2001. Other presentations are presented in this issue, pp. 317-492. 
nents on various degrees of freedom [1,2]. Chemical composition of the dc capillary glow discharge in $\mathrm{CO}_{2}-\mathrm{N}_{2}-\mathrm{He}$ gas mixture, used as an active media for the waveguide $\mathrm{CO}_{2}$ lasers, have been determined by three different methods.

\section{Methods}

In a number of cases, the method of optical actinometry permits determination of radical densities in the ground states from the emission spectra of these particles [1,3]. In the classical case, small quantities of the actinometer are added to the gas under investigation. If the radiative states of radical and actinometer are excited by a direct electron impact from their ground states, and have close to each other excitation potentials, the same group of electrons taking part in their excitation. On the basis of known experimental data, one can assume that the electron excitation cross-sections of vibronic states of electronically excited molecules proportional to the Frank-Condon factors for the relevant transitions [1]. If respective radiative states are depopulated by the radiative decay, which is a reasonable assumption for the reduced-pressure plasmas, then for the relative intensities of molecular bands (or atomic lines) of actinometer and radical, one can derive the following ratio [4]:

$$
\frac{I_{1}}{I_{2}}=\frac{N_{01}}{N_{02}} \cdot \frac{\sigma_{01}^{\max }}{\sigma_{02}^{\max }} \cdot \frac{\int_{E_{1}}^{\infty} \varphi_{01}(\in) f(\in) \epsilon^{1 / 2} d \in \sum_{E_{2}}^{\infty} \varphi_{02}^{\infty} q_{v^{0}=0}^{(1)} \psi\left(v^{0}\right) f(\epsilon) \epsilon^{1 / 2} d \in \sum_{v^{0}=0}^{\infty} q_{v^{0} v^{\prime}}^{(2)} \psi^{2}\left(v^{0}\right)}{\left.\int^{0}\right)} \cdot \frac{A_{1} \tau_{1} v_{1}}{A_{2} \tau_{2} v_{2}},
$$

where $\sigma_{0 \mathrm{i}}{ }^{\max }$ is the excitation cross-section of radiative states in maximum, $E_{i}$ is the excitation threshold, $\varphi_{0 \mathrm{i}}(\varepsilon)$ is the energy dependence of the excitation cross-section, $f(\varepsilon)$ is the electron energy distribution function, $q_{v^{\circ} v^{\prime}}$ are the Frank-Condon factors, $\psi\left(v^{0}\right)$ is the relative vibrational population of molecules in the ground state, $A_{i}, \tau_{i}$ and $v_{i}$ are the radiative transition probability, lifetime, and frequency of radiation, $N_{0 \mathrm{i}}$ is the particles absolute density in ground electronic states.

In the case of $E_{1} \sim E_{2}$ and $\varphi_{01}(\varepsilon) \sim \varphi_{02}(\varepsilon)$ via selection of radiative states, from eq 1, the absolute density of radicals can be derived in respect to the absolute density of actinometer, relative intensities of molecular bands (or atomic lines), and combination of various rate coefficients [3,4]:

$$
N_{02}=k\left(I_{1} / I_{2}\right) N_{01} \text {. }
$$

\section{Experiment}

Gas discharge has been studied in two different types of cylindrical capillary discharge tubes, of molybdenum glass and beryllium oxide. Tubes have the same length of $48 \mathrm{~mm}$ and radius of $1 \mathrm{~mm}$. The gas mixture was $\mathrm{CO}_{2}-\mathrm{N}_{2}-\mathrm{He}(1: 1: 8)$, pressure range $p=20-100$ Torr, current strength of $i=2-12 \mathrm{~mA}$, voltage between electrodes $U=2-6 \mathrm{kV}$. Spatial distribution of plasma parameters has been measured with use of a rotating quartz cube. Next to the spectroscopy, the mass spectrometry and gas chromatography have been used to measure the stable product densities in the discharges.

\section{Results and discussion}

The results of measurements of oxygen atoms and carbon oxide molecules, based on eqs 1 and 2, show that the capillary dc discharges at medium pressures are characterized by a strong dissociation of carbon dioxide molecules. Much stronger than in the discharges of classical $\mathrm{CO}_{2}$ lasers in wide $(R \sim 10 \mathrm{~mm})$ tubes. It is important to underline that molecular nitrogen, presented in the initial gas mixture, has been 
used as a natural actinometer. With increase of power input, and decrease of pressure, the dissociation degree increases monotonically. At pressure of $p=20$ Torr, and power input of $W=3 \mathrm{~W} / \mathrm{cm}$, in sealedoff regime of the discharge, the dissociation degree reaches maximum values of $\sim 80 \%$ in the molybdenum glass tube, and $\sim 60 \%$ in the beryllium oxide tube. The same tendencies were observed for the density of oxygen atoms in the discharges.

It is important to underline that the $\mathrm{CO}$ and $\mathrm{O}$ densities in the beryllium oxide tube were approximately twice as lower of the respective densities in the molybdenum glass tube (with other parameters remaining constant). This we put down to different catalytic properties of surfaces in respect of radicals recombination on the tube walls with generation of carbon dioxide molecules.

\section{RECOMBINING PLASMAS}

A typical example of the recombining plasmas is a flowing post-discharge. These types of plasmas are actively used nowadays for active treatment of the surfaces (cleaning, etching, deposition) [5,6]. In recombining plasmas, the excited states of atoms and molecules are populated by the flux directed from the recombination of ions and electrons to the ground state of stable atoms and molecules.

\section{Methods}

A particularly simple kinetic scheme can be realized for the first excited atomic states in the recombining plasmas. For example, in the conditions of an expanding cascaded arc plasmas a simple kinetic analysis shows that the density of the first excited state $n_{2}{ }^{*}$ can be found from the simple balance between the recombination flux $\Phi$ to this level and radiative decay from this level [7]:

$$
\Phi-n_{2}{ }^{*} A_{21} \Lambda_{21}=0
$$

Here, $A_{21}$ is the resonance radiative transition probability, and $\Lambda_{21}(k R)$ is the escape factor for emission of resonance radiation. The escape factor $\Lambda$ is a function of the effective optical depth $(k R)$. The functional dependence of $\Lambda_{21}(k R)$ determined by the emission and absorption line profiles [8]:

$$
k R=\frac{(\ln 2)^{1 / 2}}{4 \pi \sqrt{\pi}} \frac{\lambda_{p q}^{4}}{c \Delta \lambda_{p q}} \frac{g_{p}}{g_{q}} A_{p q} n_{q} \mu R,
$$

where $\lambda_{p q}$ is the wavelength of the radiative transition, $\Delta \lambda_{p q}$ is the width of the emission profile, $c$ is the velocity of light, $g_{p}$ and $g_{q}$ are the statistical weights, $n_{q}$ is the density of the lower state of the transition, $\mu$ is a geometrical factor [8], and $R$ is the mean radius of the plasma beam.

In the case of known recombination flux $\Phi$, and absolute density of the first excited state $n_{2}{ }^{*}$, one can determine from eq 3 the escape factor $\Lambda_{21}$ and effective optical depth $(k R)$ for the resonance radiative transition [8]. Finally, from eq 4, the particles absolute density in the ground electronic states can be derived. The method has been applied to determination of the absolute ground-state density of atomic hydrogen $\mathrm{H}(n=1)$ and $\operatorname{argon} \operatorname{Ar}\left(3 p^{6}\right)$ in a freely expanding plasma jet [9].

\section{Experiment}

Experiments have been performed for the conditions of expanded cascaded arc plasma at the Eindhoven University of Technology. Details of the experimental set-up and the measurement techniques were described earlier [9]. The hydrogen percentage in the total gas flow of $\mathrm{Ar}-\mathrm{H}_{2}$ mixture was varied in the range $0.7-10 \%$. Background pressure was $40 \mathrm{~Pa}$, gas flow rate $58 \mathrm{~cm}^{3} \mathrm{~s}^{-1}$, arc current $45 \mathrm{~A}$, arc voltage $80-115 \mathrm{~V}$. The argon density in the first excited state $\operatorname{Ar}\left(3 p^{5} 4 s\right)$ has been measured in pure argon plasmas by the absorption of eight argon spectral lines, belonging to the transition $\operatorname{Ar}\left(3 p^{5} 4 p \rightarrow 3 p^{5} 4 s\right)$. 
The hydrogen density in the first excited state $\mathrm{H}^{*}(n=2)$ has been determined in an argon-hydrogen plasma by hydrogen Balmer- $\alpha$ spectral line absorption.

\section{Results and discussion}

The radial dependencies of the $\operatorname{Ar}\left(3 p^{5} 4 s\right)$, summed over the states $3 p^{5} 4 s\left({ }^{3} P_{0},{ }^{3} P_{1},{ }^{3} P_{2},{ }^{1} \mathrm{P}_{1}\right)$, as well as of the $\mathrm{H}^{*}(n=2)$ state have been determined by absorption spectroscopy for various axial positions in the expansion both in pure argon, and in the $\mathrm{Ar}-\mathrm{H}_{2}$ gas mixtures. In [10] a similar expanding argon plasma was studied using the technique of Thomson-Rayleigh scattering. It has been shown that the agreement between two methods was quite good [9]. Hence, the assumptions upon which the discussed method is based were correct, and for the recombining expanding plasma the method can be applied for determination of ground-state density of short-lived radicals. The absolute densities of atomic hydrogen at the distances between 40 and $70 \mathrm{~mm}$ from beginning of expansion have been calculated from eq 3 . The densities were in the range $(0.2-9) \times 10^{18} \mathrm{~m}^{-3}$.

\section{CONCLUSIONS}

1. The molecular optical actinometry technique, combined with the mass spectrometry and gas chromatography methods, has been used to determine the absolute densities of the $\mathrm{O}$ atoms and $\mathrm{CO}$ molecules in the $\mathrm{CO}_{2}-\mathrm{N}_{2}-\mathrm{He}$ gas mixture of the waveguide $\mathrm{CO}_{2}$ laser. Different catalytic properties of the discharge tube materials toward recombination of radicals and association of $\mathrm{CO}_{2}$ open new possibilities for regeneration of $\mathrm{CO}_{2}$ molecules in the plasma, and as a consequence, for increasing the power of laser generation, and development of sealed-off waveguide $\mathrm{CO}_{2}$ lasers.

2. The densities of argon and hydrogen atoms in the first excited states have been determined by the technique of visible light absorption spectroscopy. Simple kinetic scheme have been employed to determine absolute ground-state density of $\mathrm{Ar}$ and $\mathrm{H}$ atoms in expanding recombining plasmas. It is important that the kinetic scheme used does not require information on the shape of the electron energy distribution function in the plasma.

3. The accuracy of presented methods of ground-state density determination is limited by the errors in the ionization, recombination, and excitation coefficients, and in the experimental signal-tonoise ratios. The proposed techniques can be used for characterization of other kinds of radicals in the chemically active plasma.

\section{REFERENCES}

1. V. M. Lelevkin, D. K. Otorbaev, D. C. Schram. Physics of Non-equilibrium Plasmas, Elsevier, Amsterdam (1992).

2. Yu. P. Raizer. Gas Discharge Physics, Springer-Verlag, Berlin (1991).

3. Zhou Qing, D. K. Otorbaev, G. J. H. Brussaard, M. C. M. van de Sanden, D. C. Schram. J. Appl. Phys. 80, 1312 (1996).

4. A. T. Baiterekov and D. K. Otorbaev. Proc. X Int. Symp. on Plasma Chemistry, 2, 2.1-26 (1991).

5. W. M. M. Kessels, R. J. Severens, A. H. M. Smets, B. A. Korevaar, G. J. Adriaenssens, D. C. Schram, M. C. M. van de Sanden. J. Appl. Phys. 89, 2404 (2001).

6. M. Gaillard, P. Raynaud, A. Ricard. Plasmas Polym. 4, 241 (1999).

7. D. K. Otorbaev, A. J. M. Buuron, N. T. Guerassimov, M. C. M. van de Sanden, D. C. Schram. J. Appl. Phys. 76, 4499 (1994).

8. T. Holstein. Phys. Rev. 83, 1159 (1951). 
9. D. K. Otorbaev, A. J. M. Buuron, M. C. M. van de Sanden, R. F. G. Meulenbroeks, D. C. Schram. J. Phys. D: Appl. Phys. 27, 1362 (1995).

10. R. F. G. Meulenbroeks, A. J. Beek, A. J. G. van Helvoort, M. C. M. van de Sanden, D. C. Schram. Phys. Rev. E 49, 4397 (1994). 